\title{
Performance of Wireless-Powered Sensor Transmission Considering Energy Cost of Sensing
}

\author{
Wanchun Liu*, Xiangyun Zhou*, Salman Durrani*, Hani Mehrpouyan ${ }^{\dagger}$, Steven D. Blostein ${ }^{\S}$ \\ ${ }^{*}$ Research School of Engineering, College of Engineering and Computer Science, The Australian National University. \\ ${ }^{\dagger}$ Department of Electrical and Computer Engineering, Boise State University. \\ ${ }^{\S}$ Department of Electrical and Computer Engineering, Queen's University. \\ Emails: \{wanchun.liu, xiangyun.zhou, salman.durrani\}@anu.edu.au, hani.mehr@ieee.org and steven.blostein@queensu.ca.
}

\begin{abstract}
Realistic modeling of energy consumption is crucial for accurate performance analysis of wireless-powered sensor nodes. In this paper, we analyze the performance of wirelesspowered sensor transmissions taking into account both the energy cost of sensing and transmission. We consider a sensor that is harvesting energy from an ambient radio-frequency (RF) signal and using this energy to perform sensing and transmission. Since energy harvesting is time-varying in nature, it introduces a delay in the sensor transmissions. We study two delay-related metrics, one measuring how frequent the sensed information is updated at the sink and the other measuring the time taken from the sensing operation to successful transmission of sensed information. We analytically characterize the statistical behavior of both metrics and find an important tradeoff between them. In particular, our results illustrate that more frequent update of sensed information at the sink increases the time taken from the sensing operation to successful transmission of sensed information.
\end{abstract}

\section{INTRODUCTION}

Energy harvesting $(\mathrm{EH})$ has been widely recognized as an enabling technology for green and self-sustainable wireless communications, especially in wireless sensor networks (WSNs) [1]. Apart from the traditional energy sources, wireless power transfer using radio-frequency (RF) signals has recently emerged as a promising approach to enable energy constrained WSNs [2-6]. Practical designs for sensor devices powered by ambient RF signals, e.g., transmitted by TV towers or macro base stations, were considered in [2], [3]. The hardware and circuit design for RF powered wireless sensor was studied in [4]. A cognitive communication protocol for wireless-powered WSN was studied in [5]. Routing and link layer protocol designs for wireless-powered WSN were studied in [6].

An important design consideration for energy-constrained wireless sensors is the modeling of energy costs. There are three major operations in wireless sensors incurring energy costs: RF transmission/reception (including idle listening [7]), information sensing (and processing), and other basic processing while active [8]. The majority of the current work on wireless sensor communications considers the energy cost of transmission, with the assumption that RF transceiver usually consumes the majority of the sensor's energy. It is usually true that the basic processing for keeping a sensor active consumes

This research was supported by the Australian Research Councils Discovery Project funding scheme (project number DP14010133). relatively less energy compared with communications. This assumption is also relevant when low-quality sensing is used. However, due to the diversity of sensors, some commonlyused sensing modules are designed with high-rate and highresolution A/D conversion and hence can be power hungry [9]. In fact, energy cost of sensing can be higher than energy cost of transmission in such scenarios (from the citations in [9]).

For EH sensors, the delay of transmission of sensed information from the sensor to the sink becomes a more critical issue than that for conventional sensors with sufficient (initial) power storage, because of the extra time required for $\mathrm{EH}$ [10], [11]. The transmission completion time was studied with several different data arrival processes in [10]. The focus in [10] was delay due to EH, while transmission was assumed to be always successful. Transmission delay was studied for a wireless-powered sensor with retransmission in [11]. Compared with conventional wireless sensors, for each (re)transmission, a certain amount of time is required for $\mathrm{EH}$ to provide enough energy for transmission, which increases the delay.

We study the performance of an energy-constrained sensor powered by ambient RF signals, e.g., signals from the nearest TV tower or macro base station, taking both energy cost of sensing and transmission into account. We consider the application scenario where a certain condition or process needs to be monitored constantly. Thus, the sensor is required to sense information and transmit it to a sink on a periodic basis [12]. Upon each successful transmission, the sink updates its recoded information. However, for a wireless-powered sensor, in order to perform the sensing and transmission, it needs to harvest enough energy from an EH process of time-varying nature. The time-varying EH process results in randomness in the delay for performing sensing and transmission. Thus, periodic update at the sink is generally impossible. Considering the fact that the EH rate from ambient RF signal is typically low, we consider a harvest-then-use protocol where the sensor performs sensing and transmission as soon as it has harvested sufficient energy. We measure delay in terms of the update cycle and successful transmission delay. The successful transmission delay metric measures the time duration from sensing to successful transmission of sensed information, while the update cycle metric measures how frequent the information is updated at the sink. Note that in conventional 
WSNs, periodic sensing protocols have a fixed update cycle [12]. In our scenario with wireless-powered sensor, the update cycle becomes a random variable due to the randomness in the amount of harvested energy. Our novel contributions are as follows:

- The delay performance of a wireless-powered EH sensor is assessed taking into account both energy cost of sensing and transmission. The conditions under which sensing and transmission occurs in order to achieve the best delay performance, are then determined.

- Two delay-related performance metrics, namely, update cycle and successful transmission delay are analyzed. Both the energy-carrying ambient RF signals and the sensorsink transmission experience time-varying fading channels, making both delay metrics random variables. The complete statistics of both metrics are derived in closed-form.

- An important tradeoff is found between the update cycle and successful transmission delay when the energy cost of sensing is comparable with or higher than the energy cost of transmission: reducing the update cycle, i.e., more frequent updates, comes at the price of longer successful transmission delay. This tradeoff is relevant when the energy cost of sensing is considered.

\section{System Model}

We consider a simple transmission scenario (shown in Fig. 1) where a sensor transmits its sensed information to the sink. The sensor is a wireless-powered node which harvests energy from the ambient RF signals. The sensor has two main functions, i.e., sensing and transmission, each having individual energy cost. In order to perform either sensing or transmission, the sensor needs to spend a certain amount of time on EH. Following current practical design of $\mathrm{EH}$ sensors [13], the sensor adopts a time-slotted or block-wise operation. We assume that one sensing operation or one transmission is performed in one time block of duration $T$. Although sensors may spend differing amounts of time on one sensing operation [14], the assumed protocol and analysis may be generalized to different sensing time durations other than $T$, which is outside the scope of this work. Considering that a sensor may perform sensing, transmission, or RF energy harvesting, we define the following types of time blocks, with associated energy costs:

- Sensing Block (SB): the sensor samples the targeted environmental information and then processes and packs sensed information into a data packet. The energy cost in a SB is denoted by $\mathcal{E}_{\mathrm{SB}}$.

- Transmission Block (TB): the sensor transmits the newest generated data packet (from the last sensing operation) to the sink with energy cost $\mathcal{E}_{\mathrm{TB}}$, i.e., the transmit power is $\mathcal{P}_{\mathrm{TB}}=\mathcal{E}_{\mathrm{TB}} / T$. If transmission is successful, we have a successful transmission block (STB); otherwise, we have a failed transmission block (FTB). We assume that successes/failures of each TB are mutually independent [11], [15]. The probability of a TB being a FTB, i.e., transmission outage, is denoted by $P_{\text {out }}$.

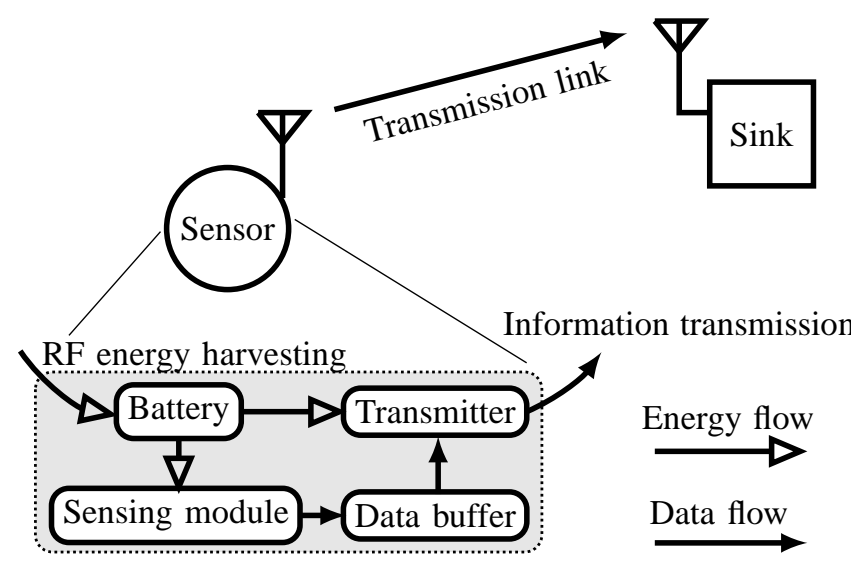

Fig. 1 System model and sensor components.

- Energy-harvesting block (EHB): the sensor harvests energy from RF signals and stores the energy in its battery. We assume that the battery has sufficient capacity such that the amount of energy stored in the battery never reaches its maximum capacity. This assumption is a reasonable since battery capacity is several joules to several thousand joules [1], while the energy level in the battery in our system is only several $\mu \mathrm{J}$ as shown in Sec. V.

\section{A. RF Energy Harvesting}

We assume the power source has a transmit power of $\mathcal{P}_{\mathrm{WPT}}$ and is located at distance $d_{\mathrm{WPT}}$ from the sensor. The wireless power transfer link experiences both large-scale path loss and small-scale Rayleigh fading, where the fading coefficient $h_{\mathrm{WPT}}$ is constant within one time block of length $T$ and varies independently from block to block. From [11], the harvested energy in each EHB is given by

$$
\mathcal{E}_{\mathrm{WPT}}\left(h_{\mathrm{WPT}}\right)=\eta T \frac{\mathcal{P}_{\mathrm{WPT}}\left|h_{\mathrm{WPT}}\right|^{2}}{d_{\mathrm{WPT}}^{\lambda}},
$$

where $\eta$ is the efficiency of RF energy harvesting plus storage of the sensor [16] and $\lambda$ is the path loss exponent. With the Rayleigh fading assumption, $\left|h_{\mathrm{WPT}}\right|^{2}$ follows an exponential distribution with unit mean, and hence, $\mathcal{E}_{\mathrm{WPT}}\left(h_{\mathrm{WPT}}\right)$ is an exponential random variable with mean $\rho$, where $\rho$ represents the average harvested energy in each EHB given by

$$
\rho=\eta T \frac{\mathcal{P}_{\mathrm{WPT}}}{d_{\mathrm{WPT}}^{\lambda}} .
$$

\section{B. Proposed Sensing and Transmission Protocol}

Considering the energy cost of sensing, it is necessary to harvest sufficient energy, $\mathcal{E}_{\mathrm{SB}}$, before sensing can occur. However, it is unwise to perform sensing as soon as the harvested energy reaches $\mathcal{E}_{\mathrm{SB}}$ because there will be insufficient energy left for transmission after the sensing operation. The time spent on EH due to insufficient energy for transmitting the sensed information results in unnecessary delay. To avoid such delay, we define the condition for the sensing operation as when the harvested energy in the battery exceeds $\mathcal{E}_{\mathrm{SB}}+\mathcal{E}_{\mathrm{TB}}$. In this way, a transmission of sensed information occurs immediately following after the sensing operation. We allow for a certain 
number of retransmissions, if transmission is not successful due to the fading channel, between the sensor and sink. Such retransmission schemes are commonly implemented in conventional WSNs [17]. However, retransmissions result in large delays. Therefore, it is important to properly adjust the number of maximum retransmissions (which should be much smaller than that of conventional sensors) and start a new sensing operation once that number is reached. We denote the maximum number of transmission as $N$, that is, a maximum of $N-1$ retransmissions.

Having the protocol for sensing and transmission operations described above, the sensor operates as follows: First, the sensor uses several EHBs to harvest enough energy, $\mathcal{E}_{\mathrm{SB}}+\mathcal{E}_{\mathrm{TB}}$, and then a $\mathrm{SB}$ and a TB occur. If the transmission in the TB is successful, i.e., having a STB, the sensor harvests energy (taking several EHBs) for the next sensing period when the battery energy exceeds $\mathcal{E}_{\mathrm{SB}}+\mathcal{E}_{\mathrm{TB}}$. If the transmission in the TB fails, i.e., having a FTB, the sensor goes back to harvesting energy (taking several EHBs) and performs a retransmission when the battery energy exceeds $\mathcal{E}_{\mathrm{TB}}$. Retransmission may occur several times until the sensed information is successfully transmitted to the sink or the maximum transmission number $N$ is reached. Then, the data packet at the sensor is dropped and the sensor goes back to harvest the energy for a new sensing operation.

Fig. 2 illustrates this process with the maximum number of transmissions set to $N=3$. The first block in Fig. 2 is a SB, followed by two FTBs (and two EHBs in between). Since the third TB is a STB, the sensed information in the first SB is successfully transmitted to the sink. Then, the sensor uses one EHB to harvest energy to conduct sensing in the next SB. After the second SB, there are three FTBs. Thus, the retransmission process is terminated after $N=3$ is reached. As a result, the sensed information in the second SB is not transmitted to the sink. Note that the time indices shown in Fig. 2 will be defined in Sec. III.

\section{Delay Related Metrics}

As described in the previous section, both sensing and (re)transmission requires a variable amount of time for $\mathrm{EH}$, which may result in significant delays in obtaining the targeted environmental information at the sink. In this section, we define two metrics to measure the delay performance of the considered sensing and transmission protocol. To enable us to define the two metrics as random variables, we first present Lemma 1.

Lemma 1. The amount of energy in the sensor's battery after each TB is an exponentially distributed random variable, $E_{o}$, with pdf:

$$
f_{E_{o}}(\epsilon)=\frac{1}{\rho} e^{-\frac{\epsilon}{\rho}},
$$

where $\rho$ is the average harvested energy in each EHB given in (1).

Proof. Because the harvested energy in each EHB, $\mathcal{E}_{\mathrm{WPT}}\left(h_{\mathrm{WPT}}\right)$ is an exponentially distributed random variable with parameter $\rho$, as we have mentioned in Sec. II.A, the energy accumulation process by consecutive EHBs can be treated as a Poisson process [18]. Using the memorylessness property of the Poisson process [18, pp. 134], conditioned on that the available energy is higher than any given threshold value, the amount of energy exceeding that threshold, $E_{o}$, has the same distribution with the harvested energy in each time block, i.e., $\mathcal{E}_{\mathrm{WPT}}\left(h_{\mathrm{WPT}}\right)$. This proof is identical to [15, Lemma 1].

For the convenience of describing the two metrics, as shown in Fig. 2, we use $t_{\mathrm{STB}, j}$ to denote the block index for the $j$ th STB during the entire sensing and transmission operation. Note that a successful transmission also means an information update at the sink. Also, it is important to associate each transmission with its information content. To this end, we use $t_{\mathrm{SB}, j}$ to denote the block index for the $\mathrm{SB}$ in which the sensed information is transmitted in the $j$ th STB. In other words, environmental information sensed at $t_{\mathrm{SB}, j}$ is successfully transmitted to the sink at $t_{\mathrm{STB}, j}$. Next, we define two delay-related metrics. For simplicity and without loss of generality, we use the number of time blocks as the delay metric.

\section{A. Successful Transmission Delay}

If the sensed information is successfully transmitted to the sink, we need to know how long it takes from sensing the information to the successful transmission of the sensed information, i.e., the successful transmission delay. A longer successful transmission delay means a more outdated information is received by the sink. For the $j$ th STB, the successful transmission delay is given by the number of time blocks from $t_{\mathrm{SB}, j}$ to $t_{\mathrm{STB}, j}$ (shown in Fig. 2). The successful transmission delay is quantified as

$$
T_{\mathrm{STD}, j}=t_{\mathrm{STB}, j}-t_{\mathrm{SB}, j}, j=1,2,3, \ldots
$$

According to the sensing and transmission protocol defined in the previous section, each SB is directly followed by a TB. From Lemma 1, the distribution of available energy after any $\mathrm{TB}$ is the same. Hence, the distribution of the available energy after the first TB following $t_{\mathrm{SB}, j}$ is the same for all $j$. Consequently, $T_{\mathrm{STD}, j} j=1,2, \ldots$, are independent and identically distributed (i.i.d.). For convenience, we remove subscript $j$ for $T_{\mathrm{STD}}$ in (3).

Note that the definition of successful transmission delay is the same as that in [10], which is the delay since a packet arrives at the source until it is successfully transmitted to the destination. But in our scenario, due to the transmission outage and an imposed maximum number of transmissions, some sensed information may not be successfully transmitted to the sink. The term successful transmission delay reflects the fact that this metric is only concerned with successful transmission of sensed information.

\section{B. Update Cycle}

Each successful transmission means an update of the sensed information held by the sink. Therefore, we are also interested 


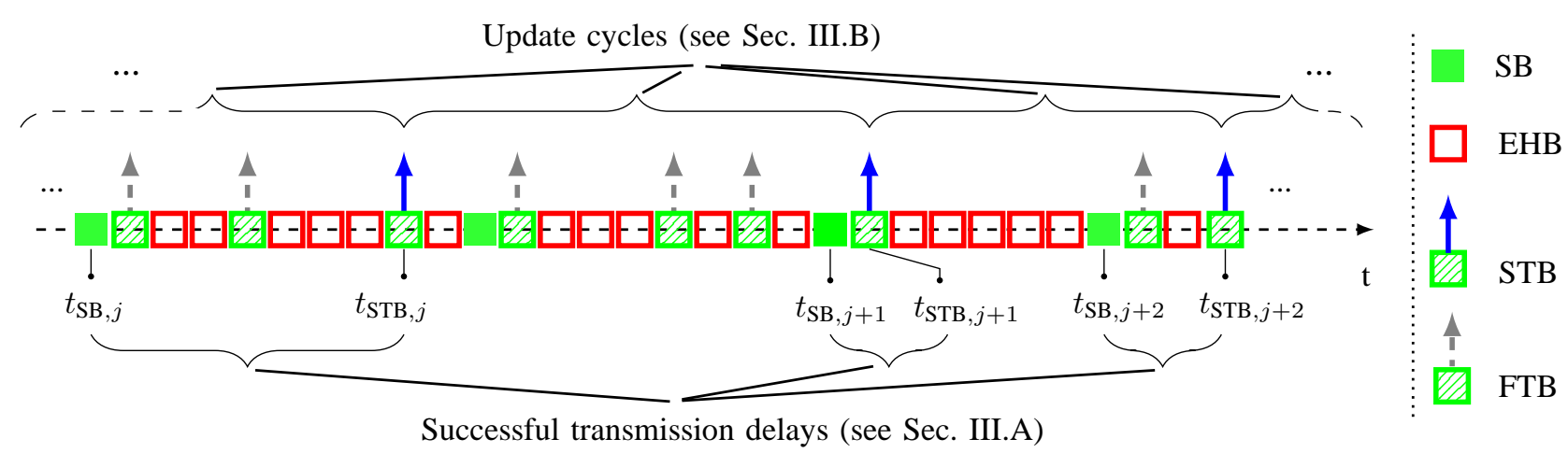

Fig. 2 Illustration of update cycle and successful transmission delay.

in measuring how often the sensed information is updated at the sink. We define the number of time blocks between two adjacent STBs as the update cycle (shown in Fig. 2). A short update cycle means that the sensed information is updated at the sink very frequently. The update cycle is defined as

$$
T_{\mathrm{UC}, j}=t_{\mathrm{STB}, j+1}-t_{\mathrm{STB}, j}, j=1,2,3, \ldots
$$

Using Lemma 1 , it is also easy to show that $T_{\mathrm{UC}, j}$ are independent and identically distributed (i.i.d.) for all $j$. For convenience, we remove the subscript of $j$ for $T_{\mathrm{UC}}$ in (4). We also use UC to denote the sequence of time blocks from an arbitrary STB to the next STB.

\section{Analytical Results}

In this section, we derive the probability mass functions (pmfs) of the delay-related metrics, $T_{\mathrm{STD}}$ and $T_{\mathrm{UC}}$ in order to complete the statistical characterization. Using these pmfs, the average values of $T_{\mathrm{STD}}$ and $T_{\mathrm{UC}}$ are derived.

\section{A. Successful Transmission Delay}

Theorem 1. The pmf of successful transmission delay is given by

$$
\begin{aligned}
& \operatorname{Pr}\left\{T_{\mathrm{STD}}=k\right\}= \\
& \sum_{n=1}^{\min \{k, N\}}\left(1-P_{\text {out }}\right)\left(P_{\text {out }}\right)^{n-1} \frac{\left(\frac{(n-1) \mathcal{E}_{\mathrm{TB}}}{\rho}\right)^{k-n} \exp \left(-\frac{(n-1) \mathcal{E}_{\mathrm{TB}}}{\rho}\right)}{\left(1-\left(P_{\text {out }}\right)^{N}\right)(k-n) !}, k \geq 1 .
\end{aligned}
$$

where $P_{\text {out }}$ and $\mathcal{E}_{\mathrm{SB}}$ are defined in Sec. II and $N$ is defined in Sec. III.

Proof. See Appendix B (which uses the definitions in Appendix A).

From (5), the average value of $T_{\mathrm{STD}}$ can be easily determined.

Corollary 1.1. The average successful transmission delay is given by

$$
\bar{T}_{\mathrm{STD}}=A_{\mathrm{suc}}+\left(A_{\mathrm{suc}}-1\right) \frac{\mathcal{E}_{\mathrm{TB}}}{\rho},
$$

where

$$
A_{\text {suc }}=\frac{N\left(P_{\text {out }}\right)^{N+1}-(N+1)\left(P_{\text {out }}\right)^{N}+1}{\left(1-\left(P_{\text {out }}\right)^{N}\right)\left(1-P_{\text {out }}\right)} .
$$

As a special case, we consider removing the constraint on the maximum number of transmissions so that all sensed information is eventually transmitted to the sink.

Corollary 1.2. $\bar{T}_{\mathrm{STD}}$ increases with $N$, and when $N \rightarrow \infty$, the asymptotic upper bound of $\bar{T}_{\mathrm{STD}}$ is given by

$$
\lim _{N \rightarrow \infty} \bar{T}_{\mathrm{STD}}=\frac{1+\frac{\mathcal{E}_{\mathrm{TB}}}{\rho} P_{\text {out }}}{1-P_{\text {out }}} .
$$

Proof. It can be shown that the derivative of $\bar{T}_{\mathrm{STD}}$ w.r.t. $N$ (treating $N$ as a continuous real number) is larger than 0 . In addition, when $N \rightarrow \infty$, we have $\left(P_{\text {out }}\right)^{N} \rightarrow$ 0 and $N\left(P_{\text {out }}\right)^{N} \rightarrow 0$. The upper bound is easily obtained.

Remark 1. From the above analytical results, we have that:

i) From Theorem 1, we know that $T_{\mathrm{STD}}$ is independent of the energy cost of sensing, $\mathcal{E}_{\mathrm{SB}},{ }^{2}$ because the delay is only affected by the energy harvesting and retransmissions that happen after the sensing operation.

ii) Allowing a larger maximum number of transmissions increases the average successful transmission delay. This might suggest that retransmissions should be avoided. However, the successful transmission delay does not take into account the cases where sensed information is not successfully transmitted to the sink. In this regard, the update cycle implicitly captures such cases.

B. Update Cycle

Theorem 2. The pmf of update cycle is given in (9), shown at the top of the next page.

Proof. See Appendix C.

Corollary 2.1. The average update cycle is given by

$$
\bar{T}_{\mathrm{UC}}=N_{E H}+\frac{\left(P_{\text {out }}\right)^{N}}{1-\left(P_{\text {out }}\right)^{N}}(N+1)+A_{\text {suc }}+1,
$$

where

$$
N_{E H}=\frac{\frac{\left(\mathcal{E}_{\mathrm{SB}}+N \mathcal{E}_{\mathrm{TB}}\right)\left(P_{\mathrm{out}}\right)^{N}}{1-\left(P_{\mathrm{out}}\right)^{N}}+\left(\mathcal{E}_{\mathrm{SB}}+A_{\mathrm{suc}} \mathcal{E}_{\mathrm{TB}}\right)}{\rho} .
$$

\footnotetext{
${ }^{2}$ This is different to the case of $T_{\mathrm{UC}}$, shown in the next page.
} 


$$
\begin{array}{r}
\operatorname{Pr}\left\{T_{\mathrm{UC}}=k\right\}=\sum_{n=1}^{\min \{k-1, N\}}\left(1-P_{\mathrm{out}}\right)\left(P_{\mathrm{out}}\right)^{n-1} \sum_{m=0}^{\left\lfloor\frac{k-n-1}{N+1}\right\rfloor}\left(P_{\mathrm{out}}\right)^{N m}\left(\frac{(1+m) \mathcal{E}_{\mathrm{SB}}+(n+N m) \mathcal{E}_{\mathrm{TB}}}{\rho}\right)^{k-(n+1)-m(N+1)} . \\
\exp \left(-\frac{(1+m) \mathcal{E}_{\mathrm{SB}}+(n+N m) \mathcal{E}_{\mathrm{TB}}}{\rho}\right) \frac{1}{(k-(n+1)-m(N+1)) !}, k \geq 2 .
\end{array}
$$

Corollary 2.2. $\bar{T}_{\mathrm{UC}}$ decreases with $N$, and when $N \rightarrow \infty$, the asymptotic lower bound of $\bar{T}_{\mathrm{UC}}$ is given by

$$
\lim _{N \rightarrow \infty} \bar{T}_{\mathrm{UC}}=\frac{1}{\rho}\left(\mathcal{E}_{\mathrm{SB}}+\mathcal{E}_{\mathrm{TB}} /\left(1-P_{\text {out }}\right)\right)+1 /\left(1-P_{\text {out }}\right)+1 .
$$

Proof. The proof is similar to Corollary 1.2, hence is omitted for brevity.

Remark 2. From the above analytical results, we have that:

i) From Theorem 2, we know that $T_{\mathrm{UC}}$ is affected by the energy cost of sensing, $\mathcal{E}_{\mathrm{SB}}$. A larger $\mathcal{E}_{\mathrm{SB}}$ means more EHBs are required to harvest a sufficient amount of energy to have a SB or multiple SBs between adjacent STBs.

ii) A larger maximum number of transmissions shorten the average update cycle, because allowing more retransmissions increases the chance of having a successful transmission. This might suggest that it is also better to increase $N$ to reduce the update cycle. But increasing $N$ also increases the successful transmission delay. Therefore, there is clearly a tradeoff between the two metrics.

\section{NuMERICAL RESUltS}

In this section, we present numerical results on the successful transmission delay and update cycle. We assume a $1 \mathrm{MW}$ TV tower [3] as the ambient RF power source at a distance of $1.71 \mathrm{~km}$ from the sensor. We set the distance between the sensor and the sink as $d=50 \mathrm{~m}$ and the noise power at the sink as $\sigma^{2}=-90 \mathrm{dBm}$ [19]. For both the power transfer link and the sensor-sink transmission link, the path loss exponent is set as $\lambda=3$ [11]. The energy conversion efficiency is set as $\eta=0.5$ [16]. The duration of a time block is $T=1$ millisecond [15]. Under such a setting, the average harvested energy at the sensor during each EHB is $\rho=0.1 \mu \mathrm{J}$ (i.e., the average received power is $0.2 \mathrm{~mW}$ and the average harvested power is $0.1 \mathrm{~mW})^{3}$. Unless otherwise stated, we set $N=10$ and $\mathcal{E}_{\mathrm{TB}}=1 \mu \mathrm{J}$ (i.e., $\left.\mathcal{P}_{\mathrm{TB}}=1 \mathrm{~mW}\right)^{4}$.

In the following, we assume that the transmission outage from the sensor to the sink occurs when the SNR at the sink $\gamma$, is lower than SNR threshold $\gamma_{0}=40 \mathrm{~dB}$ [20]. Thus, the outage probability $P_{\text {out }}$ is given by

$$
P_{\text {out }}=\operatorname{Pr}\left\{\gamma<\gamma_{0}\right\} \text {. }
$$

\footnotetext{
${ }^{3}$ Such a received power is sufficient to properly activate the RF EH circuit, e.g., a typical input power sensitivity level at the RF-DC converter is $-20 \mathrm{dBm}[16]$

${ }^{4}$ The typical transmit power of wireless sensors is several milliwatts [8].
}
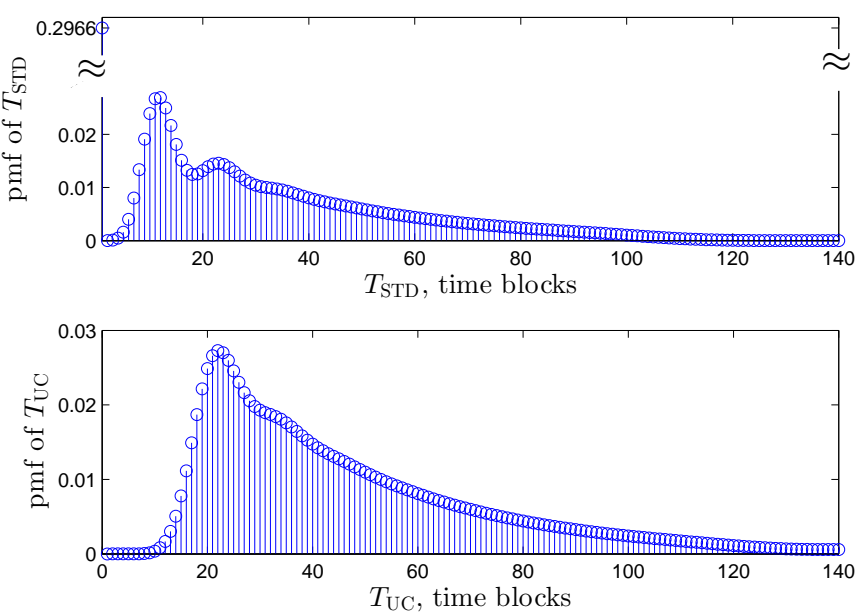

Fig. 3 pmfs for $T_{\mathrm{STD}}$ and $T_{\mathrm{UC}}$.

The SNR at the sink can be written as

$$
\gamma=\frac{|h|^{2} \mathcal{P}_{\mathrm{TB}}}{d^{\lambda} \sigma^{2}}
$$

where $\mathcal{P}_{\mathrm{TB}}=\mathcal{E}_{\mathrm{TB}} / T$, and $h$ is the source-sink channel fading. For the numerical results, we assume that $h$ is block-wise Rayleigh fading. The outage probability can then be written as

$$
P_{\text {out }}=1-\exp \left(-\frac{d^{\lambda} \sigma^{2} \gamma_{0}}{\mathcal{P}_{\mathrm{TB}}}\right)
$$

By applying (15) to the analysis in the previous section, we compute the expressions of the pmfs of $T_{\mathrm{STD}}$ and $T_{\mathrm{UC}}$ as well as their average values $\bar{T}_{\mathrm{STD}}$ and $\bar{T}_{\mathrm{UC}}$.

Fig. 3 shows the pmfs of $T_{\mathrm{STD}}$ and $T_{\mathrm{UC}}$ given in Theorems 1 and 2, respectively. Note that the pmfs generally cannot be accurately approximated by well-known distributions, such as Binomial, Geometric or Poisson.

Fig. 4 shows $\bar{T}_{\mathrm{STD}}$ and $\bar{T}_{\mathrm{UC}}$ with different $N$. We see that the average update cycle increases with energy cost of sensing, while the average successful transmission delay does not change with energy cost of sensing. As $N$ increases, $\bar{T}_{\mathrm{UC}}$ approaches its analytical lower bound Eq. (12), while $\bar{T}_{\text {STD }}$ approaches its analytical upper bound Eq. (8). Since the existing studies in the literature commonly ignore the energy cost of sensing, we also include the result with zero energy cost of sensing, i.e., setting $\mathcal{E}_{\mathrm{SB}}=0 \mu \mathrm{J}$. When $\mathcal{E}_{\mathrm{SB}}=0 \mu \mathrm{J}$, we see that $\bar{T}_{\mathrm{UC}}$ is almost constant around the value of 40 and does not change much with $N$.

Fig. 5 shows the tradeoff between $\bar{T}_{\mathrm{STD}}$ and $\bar{T}_{\mathrm{UC}}$. The different points on the same curve are achieved with different 


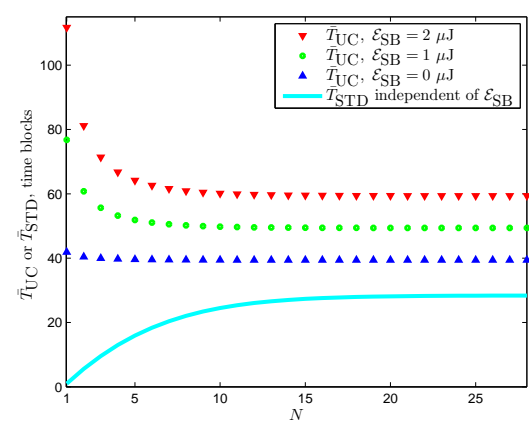

Fig. 4: $\bar{T}_{\mathrm{STD}}$ and $\bar{T}_{\mathrm{UC}}$ vs $N$.

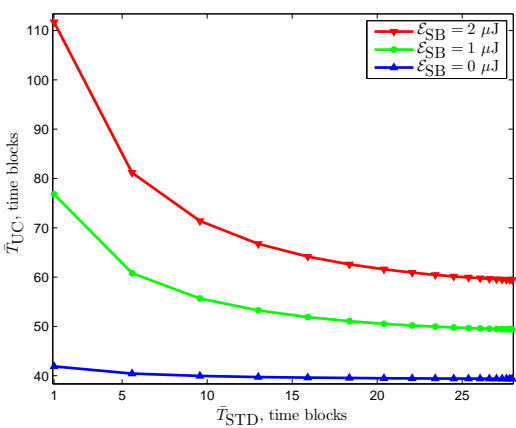

Fig. 5: Tradeoff between $\bar{T}_{\mathrm{UC}}$ and $\bar{T}_{\mathrm{STD}}$.

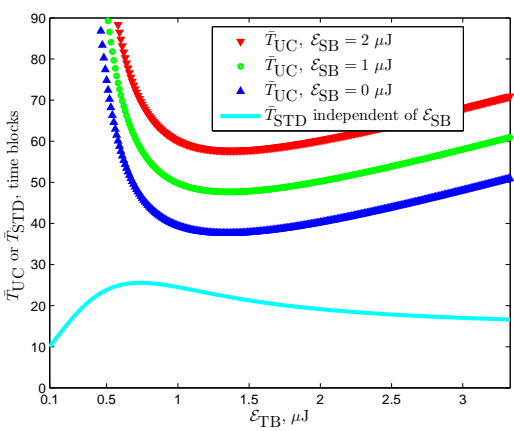

Fig. 6: $\bar{T}_{\mathrm{STD}}$ and $\bar{T}_{\mathrm{UC}}$ vs $\mathcal{E}_{\mathrm{TB}}$.
$N$. When the energy cost of sensing is comparable to or larger than the energy cost of transmission, e.g., $\mathcal{E}_{\mathrm{SB}}=1 \mu \mathrm{J}$ and $\mathcal{E}_{\mathrm{SB}}=2 \mu \mathrm{J}$, the reduction in $\bar{T}_{\mathrm{STD}}$ requires a significant increase in $\bar{T}_{\mathrm{UC}}$, and vice versa. However, when the energy cost for sensing is negligible, e.g., $\mathcal{E}_{\mathrm{SB}}=0 \mu \mathrm{J}$, such a tradeoff becomes almost unnoticeable: a significant change in $\bar{T}_{\mathrm{STD}}$ does not result in a noticeable change in $\bar{T}_{\mathrm{UC}}$. The trends in Fig. 4 and 5 are in accordance with our observations in Remarks 1 and 2.

Fig. 6 shows how $\bar{T}_{\mathrm{STD}}$ and $\bar{T}_{\mathrm{UC}}$ change with the energy cost of transmission $\mathcal{E}_{\mathrm{TB}}$ (or effectively the transmit power). We see that $\bar{T}_{\mathrm{UC}}$ first decreases and then increases with increasing transmit power. This is as expected: if the transmit power is very small, the $P_{\text {out }}$ is very high, resulting in a large number of retransmissions until the sensed information is successfully transmitted or $N=10$ is reached. As a result, $\bar{T}_{\mathrm{UC}}$ is large when the transmit power is small. If the transmit power is too large, a significant amount of time is spent on charging the sensor in order to reach the required transmit power, thus $\bar{T}_{\mathrm{UC}}$ is large as well. In contrast with $\bar{T}_{\mathrm{UC}}$, we see that $\bar{T}_{\mathrm{STD}}$ first increases and then decreases with increasing transmit power. Firstly, the number of transmissions cannot be larger than $N$. If the transmit power is almost zero, not much time is needed to charge the sensor to perform all the necessary (re)transmissions. Hence, $\bar{T}_{\mathrm{STD}}$ is very small with sufficiently small transmit power. When the transmit power is very large, the probability of successful transmission is very high, i.e., the occurrence of $T_{\mathrm{STD}}=1$ is often, and thus $\bar{T}_{\mathrm{STD}}$ is small.

\section{CONCLUSIONS}

In this work, we investigated two delay-related metrics for transmission of information with energy harvesting, taking into account the energy costs of both sensing and transmission. We illustrated the tradeoff between successful transmission delay and update cycle, which is an important design consideration when the energy cost of sensing is comparable to the energy cost of transmission. Closed-form expressions were derived for both metrics which can be used to assist the transmission design of future wireless-powered sensor networks.

\section{ApPENDix A: EVEnt DEFINITIONS}

To assist the proofs of the main results, we define several events (according to [18]) for convenience:

- $\Lambda_{\mathrm{SB}-\mathrm{FTBs}}$ : Given a SB, it is followed by $N$ FTBs, i.e., all the $N$ transmissions are failed.

- $\Lambda_{\mathrm{SB}-\mathrm{STB}, i}, 1 \leq i \leq N$ : Given a $\mathrm{SB}$, it is followed by $i$ TBs before the next SB, i.e., the first $i-1$ TBs are FTBs, while the last TB is a STB.

- $\Lambda_{\mathrm{EHB}, i, \epsilon}: i$ EHBs occur after a TB to make the available energy no less than $\epsilon$.

From the definitions, we know that $\Lambda_{\mathrm{SB}-\text { FTBs }}$ and $\Lambda_{\mathrm{SB}-\mathrm{STB}, i}$ are mutually exclusive events, and they collectively complete the set of all possible sequences of TBs after a SB before the next SB occurs. Thus, the union set of $\Lambda_{\mathrm{SB}-\mathrm{STB}, i}$, for $i=1,2, \ldots, N$, is denoted as $\bar{\Lambda}_{\mathrm{SB}-\mathrm{FTBs}}$ which is the complementary set of $\Lambda_{\mathrm{SB}-\mathrm{FTBs}}$. Also we have

$$
\begin{aligned}
& \operatorname{Pr}\left\{\Lambda_{\mathrm{SB}-\mathrm{FTBs}}\right\}=\left(P_{\text {out }}\right)^{N} \\
& \operatorname{Pr}\left\{\Lambda_{\mathrm{SB}-\mathrm{STB}, i}\right\}=\left(P_{\text {out }}\right)^{i-1}\left(1-P_{\text {out }}\right)=\left(P_{\text {out }}\right)^{i-1}\left(1-P_{\text {out }}\right) .
\end{aligned}
$$

From Lemma 1 and its proof, we know that the energy accumulation process during EHBs after a TB is a Poisson process, thus, we have

$$
\operatorname{Pr}\left\{\Lambda_{\mathrm{EHB}, i, \epsilon}\right\}=\frac{(\epsilon / \rho)^{i} e^{-\epsilon / \rho}}{i !}, i=0,1,2, \ldots
$$

\section{APPENDIX B: Proof OF THEOREM 1}

The definition of $T_{\mathrm{STD}}$ implies that one of the events $\Lambda_{\mathrm{SB}-\mathrm{STB}, i}$, for $i=1,2, \ldots, N$, occur, i.e., $\bar{\Lambda}_{\mathrm{SB}-\mathrm{FTBs}}$ occurs. Thus, we have

$$
\operatorname{Pr}\left\{T_{\mathrm{STD}}=k\right\}=\operatorname{Pr}\left\{T_{\mathrm{STD}}=k \mid \bar{\Lambda}_{\mathrm{SB}-\mathrm{FTBS}}\right\} .
$$

From the law of total probability

$$
\begin{aligned}
& \operatorname{Pr}\left\{T_{\mathrm{STD}}=k\right\}=\operatorname{Pr}\left\{T_{\mathrm{STD}}=k \mid \bar{\Lambda}_{\mathrm{SB}-\mathrm{FTB}}\right\} \\
& =\sum_{n} \operatorname{Pr}\left\{T_{\mathrm{STD}}=k \mid \Lambda_{\mathrm{SB}-\mathrm{STB}, n}, \bar{\Lambda}_{\mathrm{SB}-\mathrm{FTB}}\right\} . \\
& \quad \operatorname{Pr}\left\{\Lambda_{\mathrm{SB}-\mathrm{STB}, n} \mid \bar{\Lambda}_{\mathrm{SB}-\mathrm{FTBs}}\right\}, \\
& \stackrel{(a)}{=} \sum_{n} \operatorname{Pr}\left\{T_{\mathrm{STD}}=k \mid \Lambda_{\mathrm{SB}-\mathrm{STB}, n}\right\} \operatorname{Pr}\left\{\Lambda_{\mathrm{SB}-\mathrm{STB}, n} \mid \bar{\Lambda}_{\mathrm{SB}-\mathrm{FTBs}}\right\},
\end{aligned}
$$

where $n$ represents the number of TBs during $T_{\mathrm{STD}}$ blocks, and $(a)$ is true since $\Lambda_{\mathrm{SB}-\mathrm{STB}, n}$ is a subset of $\Lambda_{\mathrm{SB}-\mathrm{FTBs}}$. It is easy to see that when $n>k, \operatorname{Pr}\left\{T_{\mathrm{STD}}=k \mid \Lambda_{\mathrm{SB}-\mathrm{STB}, n}\right\}=0$. 
When $n>N, \operatorname{Pr}\left\{\Lambda_{\mathrm{SB}-\mathrm{STB}, n} \mid \bar{\Lambda}_{\mathrm{SB}-\mathrm{FTBs}}\right\}=0$. Thus, in (20), the summation should be taken from $n=1$ to $\min \{k, N\}$. Thus, in $k$ time blocks, except for $n$ TBs, there are $k-n$ EHBs to harvest energy for conducting $n-1$ TBs. The first TB happens directly after the SB, hence, does not require extra EHBs. From (18), the conditional probability in (20) is given by

$$
\begin{array}{r}
\operatorname{Pr}\left\{T_{\mathrm{STD}}=k \mid \Lambda_{\mathrm{SB}-\mathrm{STB}, n}\right\}=\operatorname{Pr}\left\{\Lambda_{\left.\mathrm{EHB}, k-n,(n-1) \mathcal{E}_{\mathrm{TB}}\right\}}\right. \\
=\frac{\left(\frac{(n-1) \mathcal{E}_{\mathrm{TB}}}{\rho}\right)^{k-n} \exp \left(-\frac{(n-1) \mathcal{E}_{\mathrm{TB}}}{\rho}\right)}{(k-n) !} .
\end{array}
$$

From (16) and (17), we have

$$
\operatorname{Pr}\left\{\Lambda_{\mathrm{SB}-\mathrm{STB}, n} \mid \bar{\Lambda}_{\mathrm{SB}-\mathrm{FTBs}}\right\}=\frac{\left(P_{\text {out }}\right)^{n-1}\left(1-P_{\text {out }}\right)}{1-\left(P_{\text {out }}\right)^{N}} .
$$

By taking (21) and (22) into (20), we obtain the pmf of $T_{\mathrm{STD}}$ given in Theorem 1 .

\section{APPENDIX C: PROOF OF THEOREM 2}

During a UC of $T_{\mathrm{UC}}$ time blocks, zero or several consecutive $\bar{\Lambda}_{\mathrm{SB}-\mathrm{FTBs}}$ happens first, then a $\Lambda_{\mathrm{SB}-\mathrm{STB}, i}, 1 \leq i \leq N$ happens. In other words, for all the SBs in a UC, only the last one is followed by a $\bar{\Lambda}_{\mathrm{SB}-\mathrm{FTBs}}$ (successful transmission for the sensed information), while all the previous SBs are followed by $\Lambda_{\mathrm{SB}-\mathrm{FTBs}}$ (all the transmissions are failed). From the law of total probability, we have

$$
\begin{aligned}
& \operatorname{Pr}\left\{T_{\mathrm{UC}}=k\right\} \\
& =\sum_{n} \sum_{m} \operatorname{Pr}\left\{T_{\mathrm{UC}}=k \mid m \bar{\Lambda}_{\mathrm{SB}-\mathrm{FTBs}}\right. \text { followed by }
\end{aligned}
$$$$
\text { a } \Lambda_{\mathrm{SB}-\mathrm{STB}, n} \text { in the UC\} }
$$

- $\operatorname{Pr}\left\{\bar{\Lambda}_{\mathrm{SB}-\mathrm{FTBs}}\right.$ happens $m$ times, then a $\Lambda_{\mathrm{SB}-\mathrm{STB}, n}$ happens $\}$,

where $m$ represents the number of $\Lambda_{\mathrm{SB}-\mathrm{FTBs}}$ in the UC. From the definition of $T_{\mathrm{UC}}$, an update cycle should at least include one SB and one TB. Thus, in (23),

$$
k \geq 2, n+1 \leq k \text { and } n \leq N .
$$

Because a $\Lambda_{\mathrm{SB}-\mathrm{FTBs}}$ contains $1 \mathrm{SB}$ and $N$ TBs (at least $N+1$ time blocks), and a $\Lambda_{\mathrm{SB}-\mathrm{STB}, n}$ contains $1 \mathrm{SB}$ and $n-1 \mathrm{TBs}$ (at least $n+1$ time blocks), the number of $\Lambda_{\mathrm{SB}-\mathrm{FTBs}}$ in the UC with $k$ time blocks should satisfy

$$
0 \leq m \leq\left\lfloor\frac{k-(n+1)}{N+1}\right\rfloor .
$$

Thus, for the $k$ time blocks in the UC, except for $m+1$ SBs and $n+N m$ TBs, there are $k-(n+1)-m(N+1)$ EHBs to harvest enough energy for the aforementioned SBs and TBs. From (18), the conditional probability in (23) is given by

$$
\begin{gathered}
\operatorname{Pr}\left\{T_{\mathrm{UC}}=k \mid m \bar{\Lambda}_{\mathrm{SB}-\mathrm{FTBs}} \text { followed by a } \Lambda_{\mathrm{SB}-\mathrm{STB}, n} \text { in the UC }\right\} \\
=\operatorname{Pr}\left\{\Lambda_{\left.\mathrm{EHB}, k-(n+1)-m(N+1),(1+m) \mathcal{E}_{\mathrm{SB}}+(n+N m) \mathcal{E}_{\mathrm{TB}}\right\}}\right. \\
=\left(\frac{(1+m) \mathcal{E}_{\mathrm{SB}}+(n+N m) \mathcal{E}_{\mathrm{TB}}}{\rho}\right)^{k-(n+1)-m(N+1)} \\
\cdot \frac{\exp \left(-\frac{(1+m) \mathcal{E}_{\mathrm{SB}}+(n+N m) \mathcal{E}_{\mathrm{TB}}}{\rho}\right)}{(k-(n+1)-m(N+1)) !} .
\end{gathered}
$$

Since the probabilities of $\Lambda_{\mathrm{SB}-\mathrm{FTBs}}$ and $\Lambda_{\mathrm{SB}-\mathrm{STB}, n}$ do not change with time (see Lemma 1 and Appendix A), we have

$\operatorname{Pr}\left\{\bar{\Lambda}_{\mathrm{SB}-\mathrm{FTBs}}\right.$ happens $m$ times, then a $\Lambda_{\mathrm{SB}-\mathrm{STB}, n}$ happens $\}$ $=\operatorname{Pr}\left\{\Lambda_{\mathrm{SB}-\mathrm{FTBs}}\right\}^{m} \operatorname{Pr}\left\{\Lambda_{\mathrm{SB}-\mathrm{STB}, n}\right\}=\operatorname{Pr}\left\{\Lambda_{\mathrm{SB}-\mathrm{FTBs}}\right\}^{m}$.

Therefore, by taking (16), (17), (27), (26), (25) and (24) into (23), the pmf of $T_{\mathrm{UC}}$ is given in Theorem 2 .

\section{REFERENCES}

[1] S. Sudevalayam and P. Kulkarni, "Energy harvesting sensor nodes: Survey and implications," IEEE Commun. Surveys Tuts., vol. 13, no. 3, pp. 443-461, Third Quarter 2011.

[2] R. Shigeta, T. Sasaki, D. M. Quan, Y. Kawahara, R. Vyas, M. Tentzeris, and T. Asami, "Ambient RF energy harvesting sensor device with capacitor-leakage-aware duty cycle control," IEEE Sensors J., vol. 13, no. 8, pp. 2973-2983, Aug. 2013.

[3] A. Parks, A. Sample, Y. Zhao, and J. Smith, "A wireless sensing platform utilizing ambient RF energy," in Proc. IEEE RWS, Jan. 2013, pp. 331333.

[4] Z. Popovic, E. Falkenstein, D. Costinett, and R. Zane, "Low-power farfield wireless powering for wireless sensors," Proc. IEEE, vol. 101, no. 6, pp. 1397-1409, Jun. 2013.

[5] S. Lee, R. Zhang, and K. Huang, "Opportunistic wireless energy harvesting in cognitive radio networks," IEEE Trans. Wireless Commun., vol. 12, no. 9, pp. 4788-4799, Sep. 2013.

[6] R. Doost, K. Chowdhury, and M. Di Felice, "Routing and link layer protocol design for sensor networks with wireless energy transfer," in Proc. IEEE GLOBECOM, Dec. 2010, pp. 1-5.

[7] A. Abrardo, L. Balucanti, and A. Mecocci, "A game theory distributed approach for energy optimization in WSNs," ACM Trans. Sen. Netw., vol. 9, no. 4, pp. 44:1-44:22, Jul. 2013.

[8] V. Gungor and G. Hancke, "Industrial wireless sensor networks: Challenges, design principles, and technical approaches," IEEE Trans. Ind. Electron., vol. 56, no. 10, pp. 4258-4265, Oct. 2009.

[9] V. Raghunathan, S. Ganeriwal, and M. Srivastava, "Emerging techniques for long lived wireless sensor networks," IEEE Commun. Mag., vol. 44, no. 4, pp. 108-114, Apr. 2006.

[10] J. Yang and S. Ulukus, "Transmission completion time minimization in an energy harvesting system," in Proc. IEEE CISS, Mar. 2010, pp. 1-6.

[11] T. Wu and H.-C. Yang, "On the performance of overlaid wireless sensor transmission with RF energy harvesting," IEEE J. Sel. Areas Commun., vol. 33, no. 8, pp. 1693-1705, Aug. 2015.

[12] S. Rhee, D. Seetharam, and S. Liu, "Techniques for minimizing power consumption in low data-rate wireless sensor networks," in Proc. IEEE WCNC, vol. 3, Mar. 2004, pp. 1727-1731.

[13] P. Lee, Z. A. Eu, M. Han, and H. Tan, "Empirical modeling of a solar-powered energy harvesting wireless sensor node for time-slotted operation," in Proc. IEEE WCNC, Mar. 2011, pp. 179-184.

[14] V. Raghunathan, S. Ganeriwal, and M. Srivastava, "Emerging techniques for long lived wireless sensor networks," IEEE Commun. Mag., vol. 44, no. 4, pp. 108-114, Apr. 2006.

[15] A. A. Nasir, X. Zhou, S. Durrani, and R. A. Kennedy, "Wirelesspowered relays in cooperative communications: Time-switching relaying protocols and throughput analysis," IEEE Trans. Commun., vol. 63, no. 5, pp. 1607-1622, May 2015.

[16] L. Xiao, P. Wang, D. Niyato, D. Kim, and Z. Han, "Wireless networks with RF energy harvesting: A contemporary survey," IEEE Commun. Surveys Tuts., vol. 17, no. 2, pp. 757-789, Second Quarter 2015.

[17] C. Wang, K. Sohraby, B. Li, M. Daneshmand, and Y. Hu, "A survey of transport protocols for wireless sensor networks," IEEE Netw., vol. 20, no. 3, pp. 34-40, May 2006.

[18] R. Durrett, Probability: Theory and Examples. Cambridge University Press, 2010.

[19] I. Flint, X. Lu, N. Privault, D. Niyato, and P. Wang, "Performance analysis of ambient RF energy harvesting: A stochastic geometry approach," in Proc. IEEE GLOBECOM, Dec. 2014, pp. 1448-1453.

[20] Z. Ding, S. Perlaza, I. Esnaola, and H. V. Poor, "Power allocation strategies in energy harvesting wireless cooperative networks," IEEE Trans. Wireless Commun., vol. 13, no. 2, pp. 846-860, Feb. 2014. 